\title{
PREPARACIÓN EN NORMAS INTERNACIONALES DE INFORMACIÓN FINANCIERA EN LAS PYMES DE VILLAVICENCIO - COLOMBIA ${ }^{1}$
}

\author{
PREPARATION IN INTERNATIONAL FINANCIAL REPORTING STANDARDS IN SMES \\ VILLAVICENCIO - COLOMBIA
}

\section{PREPARAÇÃO NAS NORMAS INTERNACIONAIS DE RELATO FINANCEIRO EM PME EM VILLAVICENCIO - COLOMBIA}

\author{
Por: RUÍZ SÁNCHEZ _ María del Carmen
}

Contadora Pública, Universidad Santiago de Cali. Magister en Administración, Instituto Tecnológico de Monterrey. Docente investigadora, Universidad de los Llanos, Villavicencio. Coordinadora del grupo de investigación GI Financiero. Email: mariacruiz@unillanos.edu.co, Colombia.

DOI: http://dx.doi.org/10.22267/rtend.171802.74

\begin{abstract}
RESUMEN
El proceso de convergencia contable hacia estándares internacionales, en Colombia, se convirtió en todo un reto para las pequeñas y medianas entidades (Pymes), debido a la reciente normatividad que las rige, a la escasez de estudios que sirvan de guía y a grandes limitaciones de recursos: financieros, tecnológicos y humanos para su implementación. Este artículo presenta los resultados finales de un proyecto de investigación, que busca establecer los efectos del nivel de preparación en Normas Internacionales de Información Financiera (NIIF) en su proceso de implementación, en las Pymes de Villavicencio. Se describen las características y evolución del proceso en una muestra de 34 Pymes. Los resultados indican que la mayoría de las Pymes no iniciaron el proceso, de acuerdo con el cronograma fijado para ello y, las que lo hicieron, evidencian grandes debilidades en la preparación que generan retrasos en su desarrollo; igualmente, se proponen múltiples interrogantes, que con seguridad, se constituirán en insumos para futuros estudios.
\end{abstract}

1 Artículo producto del proyecto de investigación "Efectos de la preparación en Normas Internacionales de Información Financiera en las Pymes de Villavicencio", realizado por el grupo de investigación GI Financiero, adscrito al programa de Contaduría Pública de la Universidad de los Llanos, para participar en la Convocatoria Docentes de Planta 002 P 2014. 
Palabras clave: convergencia contable, NIIF, pyme, preparación.

JEL: M41.

\begin{abstract}
The process of accounting convergence to international standards, in Colombia, became a challenge for small and medium-sized entities (SMEs), due to the recent regulations governing it, constraints of resources and paucity of studies that serve as a guide: financial, technological and human development. This article presents the results of a research project, which seeks to establish the effects of the level of preparedness in international of financial reporting standards (IFRS) in its process of implementation, in the SMEs of Villavicencio; describes the characteristics and evolution of the process in a sample of 34 SMEs. The results indicate that the majority of SMEs did not start the process, according to the schedule set for this and, which did, shows great weaknesses in the preparation which generate delays in its development; Similarly, proposes multiple questions arise, which certainly will constitute inputs for future studies.
\end{abstract}

Keywords: accounting convergence, SME, training.

JEL: M41.

\title{
RESUMO
}

O processo de convergência contábil aos padrões internacionais, na Colômbia, tornou-se um desafio para as pequenas e médias (PMEs) devido às regulamentações recentes que o rege, a escassez de estudos para orientar e grandes limitações recursos: recursos financeiros, tecnológicos e humanos para o seu desenvolvimento. Este artigo apresenta os resultados finais de um projeto de pesquisa que procura estabelecer os efeitos do nível de preparação em International Financial Reporting Standards (IFRS) no seu processo de implementação em PME em Villavicencio. as características e evolução do processo em uma amostra de 34 PME. Os resultados indicam que a maioria das PME não iniciar o processo, de acordo com o cronograma estabelecido para ele; Além disso, levantou muitas questões, o que certamente irá constituir factores de produção para estudos futuros.

Palavras-chave: convergência contábil, IFRS, SME, preparação.

JEL: M41

\section{INTRODUCCIÓN}

La contabilidad colombiana vive una coyuntura especial, en la búsqueda de utilizar un criterio más internacional en el procesamiento de la información y presentación de informes, se insertó, formalmente, en el marco normativo de las Normas Internacionales de Información Financiera (NIIF), con la Ley 1314 de 2009, por la cual se regulan los principios y normas de contabilidad e información financiera y de aseguramiento de la información. Sin embargo, solo a finales del año 2012 empezó a expedirse la normatividad 
que regula los procesos para su implementación, en dos de los tres grupos preparadores de información: Las grandes empresas que conforman el grupo uno, reguladas por el Decreto 2784 de 2012, y las microempresas pertenecientes al: grupo tres, regidas por el Decreto 2706 de 2012; el resto fueron incluidas en el grupo dos, que corresponde a las pequeñas y medianas empresas (Pymes), para las cuales se expidió el Decreto 3022 en Diciembre de 2013.

Las citadas normas, fijaron el cronograma que debía seguirse y, en éste, se incluyó la preparación obligatoria como parte de las actividades de la implementación. En tal sentido, en este artículo se presentan los resultados de un proyecto de investigación que tiene por objeto establecer los efectos del nivel de preparación e implementación de las NIIF, en las Pymes de Villavicencio.

Aunque la contabilidad tiene múltiples definiciones, para efectos del presente documento, se concibe la contabilidad “como una ciencia de carácter económico" (Cañibano, 1975. Citado por Tua, 1988: 15). En atención a que las NIIF orientan sus objetivos hacia la satisfacción de las necesidades de información a una amplia gama de usuarios y, el grado de utilidad que generan se constituye en factor determinante para evaluar su capacidad de predicción, se articulan con la teoría o paradigma de la utilidad, también denominada escuela de la utilidad "que pretende y persigue proporcionar la mayor utilidad posible en la toma de decisiones, a la vez que los criterios tradicionales de verificabilidad y objetividad" (Tua, 1995: 23).

Vale la pena resaltar que las Pymes colombianas, caracterizadas por múltiples dificultades y escasos recursos, se ven abocadas a un cambio de paradigma en la elaboración de su información contable y financiera, de una contabilidad financiera bajo el marco de principios de contabilidad generalmente aceptados, que otorga prevalencia al control sobre la predicción (Tua, 2009), al costo histórico para valorar los hechos económicos (Ferrer, 2013) y, marcada incidencia tributaria (Zevallos, 2007; Ferrer, 2013) deben pasar a presentar estados financieros completos, fiables y transparentes, que satisfagan las necesidades de los usuarios externos de la información de acuerdo con los preceptos de las NIIF. Panorama que se agrava al tener en cuenta que dichas necesidades, giran en torno al comportamiento de las situaciones en el mediano y largo plazo, y al análisis de diversos escenarios, en el cual se requiere de la plena eficiencia en el uso de los recursos y elevados conocimientos en el manejo de las citadas normas, situación que se ha resaltado en dos de los escasos estudios sobre implementación de las NIIF en las Pymes colombianas, Salazar (2013) y Cardona, Velandia \& Giraldo (2013).

Para determinar el estado de los procesos de implementación de las NIIF en las Pymes de Villavicencio, el estudio partió de las actividades definidas por la Superintendencia de Sociedades para elaborar el Estado de Situación Financiera de Apertura (ESFA) por parte de las entidades del grupo dos. Éstas se agruparon en tres etapas de acuerdo al orden en que deben llevarse a cabo: preparación, desarrollo y resultados. Igualmente, cada etapa se ha relacionado con un nivel o grado de avance de los procesos, con base en dos parámetros: 
- Fase en que se encuentra el proceso de implementación.

- Cumplimiento del cronograma previsto por el Decreto 3022 de 2013, aplicable al grupo dos.

Para efectos de asignar el nivel de desarrollo, la etapa de preparación se relaciona con una ejecución del proceso en nivel bajo, la etapa de desarrollo con un nivel medio y la de resultados con un nivel alto.

El procesamiento y análisis de la información se realizó con el apoyo de dos sistemas informáticos: Excel de Microsoft 2010, para la organización de la información y el software IBM SPSS Statistics versión 19, para el procesamiento y generación de los índices estadísticos.

La estructura seguida en el artículo, se compone de cinco apartados; el primero presenta el contexto teórico que soporta el estudio, el segundo, expone la metodología observada, el tercero explica los resultados obtenidos, el cuarto ilustra la discusión de los resultados con los referentes y, finalmente, en el quinto se disponen las conclusiones.

\section{MARCO TEÓRICO}

El estudio de las Pymes ha ganado terreno en las últimas décadas, debido a la creciente participación en el tejido empresarial de los diferentes países, especialmente de América Latina, se resalta que "su creciente importancia en los procesos de desarrollo económico de los países de la región se debe fundamentalmente al empleo que generan, sobre todo en el ámbito urbano" (Zevallos, 2007: 1), y aporte a la economía de los países como lo expone Saavedra (2012: 98) "La relevancia económica (aporte al PIB) no es el único parámetro para dimensionar la importancia de la pyme, es necesario también considerar el papel de carácter social que juegan las mismas, al promover el autoempleo".

Vale la pena destacar que las Pymes evidencian características específicas que pueden limitar o posibilitar su desarrollo; algunos aspectos afectan su desempeño, a nivel Latinoamericano pueden mencionarse "el clima de negocios y la estructura productiva, incluyendo factores como el acceso al financiamiento, a las tecnologías, los recursos humanos y la existencia de sistemas de articulación productiva, entre otros" (CEPAL-OCDE,, 2013: 52), aunque también evidencian habilidad para ajustarse al entorno en que operan, de tal forma que su información contable se ajusta a las condiciones y requerimientos propios de la organización (Sánchez y Giraldo, 2008); en tal sentido, toma gran importancia el objetivo de la convergencia hacia las normas internacionales para mejorar su productividad.

A pesar de su importancia, una de las dificultades para el estudio de las Pymes es la gran diversidad de aspectos definidos; en Colombia, dicha clasificación se realiza con base en dos parámetros, de acuerdo con lo previsto en la Ley 590 de 2000, modificada por la Ley 905 de 2004, éstos son: el valor total de los activos, expresado en términos del salario mínimo mensual legal vigente (SMMLV) y la planta de personal según el número de trabajadores, como se presenta en la siguiente tabla: 
Tabla 1. Tamaño de las Pyme en Colombia

\begin{tabular}{|c|c|c|}
\hline $\begin{array}{c}\text { Tamaño de la } \\
\text { Empresa }\end{array}$ & Valor Total de Activos en SMMLV & $\begin{array}{c}\text { Planta de personal según } \\
\text { Número de Trabajadores }\end{array}$ \\
\hline Mediana & 5.001 a 30.000 & Entre 51 y 200 \\
\hline Pequeña & 501 y menor a 5000 & Entre 11 y 50 \\
\hline
\end{tabular}

Fuente. Elaboración propia a partir de Ley 905 de 2004

\section{Las NIIF en la ciencia contable}

El desarrollo de la ciencia contable se relaciona con la evolución misma de la sociedad, aunque a lo largo de la historia se le atribuyó un carácter meramente económico, debido a sus vínculos directos con el modo de producción regente, al cual debe en buena parte sus cambios y posibilidades de desarrollo y, con la actividad económica informada; en la coyuntura actual se le vincula con el accionar de las unidades económicas y su responsabilidad social, por ende también se visualiza a la contabilidad como una ciencia de carácter social. Estos planteamientos hacen que estudiosos como Mattessich (citado por Mejía, 2005: 135) le asignen a la aplicabilidad del conocimiento contable un carácter de "realidad económico-social".

Aunque la contabilidad tiene múltiples definiciones, para efectos del presente documento, se concibe la contabilidad como una ciencia de carácter económico, cuyo objeto es el conocimiento pasado, presente y futuro de la realidad económica, en términos cuantitativos a todos sus niveles organizativos (Cañibano, 1975; Citado por Tua, 1988: 15)

Como toda ciencia, se fundamenta en una serie de teorías, modelos, principios y paradigmas; las normas internacionales de información financiera - NIIF emitidas por el Consejo de Normas Internacionales de Contabilidad (IASB, por sus siglas en inglés), se generalizaron en las dos últimas décadas como el modelo a seguir, en la búsqueda de imprimir el mayor grado de homogeneidad a la presentación de informes de propósito general por parte de las empresas.

Las NIIF orientan sus objetivos hacia la satisfacción de las necesidades de información de una amplia gama de usuarios y, el grado de utilidad que generan se constituye en factor determinante para evaluar su capacidad de predicción. En tal sentido, se articulan con la teoría o paradigma de la utilidad, también denominada escuela de la utilidad, "que pretende y persigue proporcionar la mayor utilidad posible en la toma de decisiones, a la vez que los criterios tradicionales de verificabilidad y objetividad" (Tua, 1995: 23).

Debido al contexto cambiante en que se encuentra inmersa la contabilidad, los conceptos que la sustentan han sufrido modificaciones, por tanto, sus procesos y resultados, dinámica que a su vez impone nuevas y mayores exigencias, lo que hace que la coyuntura actual se oriente hacia la predicción y, reemplace el tradicional objetivo del control (Tua, 2009). 
La implementación de las NIIF por parte de las Pymes representa un escenario de grandes cambios, al pasar del modelo de principios de contabilidad generalmente aceptados al de normas internacionales de información financiera, que conlleva modificaciones sustanciales del orden conceptual, de aplicación práctica, reestructuración de procesos contables y financieros, y la determinación de los impactos generados por la implementación de dichos cambios, entre otros, el registro de las operaciones con base en las NIIF requiere definir políticas y estrategias coherentes con la línea de negocio, como lo expone Gonzalo (2004: 110) "van desde la delimitación de los segmentos informados y la clasificación de los valores según la finalidad para la que se tengan en la empresa, debido a que cada finalidad exige una contabilización diferente".

En términos generales, respecto del proceso de adopción y/o convergencia hacia las NIIF merece resaltarse, que la aplicación por primera vez es considerado uno de los momentos de mayor trascendencia del proceso de implementación, debido a que en él se requieren de múltiples interpretaciones que deben tomarse con base en la línea de negocio; en palabras de Vásquez y Franco (2014: 3) "el modelo de negocio orienta el impacto contable y financiero de la empresa, como inductor de los efectos sobre los sistemas de información, sus procesos y políticas financieras" por tanto el conocimiento del mismo se constituye en elemento fundamental para el desarrollo del proceso de implementación de las NIIF.

Por lo anterior, es imperativo que las Pymes se fortalezcan en las competencias requeridas para la aplicación de las NIIF, que les permita mejorar aspectos tales como: identificar los roles de los diferentes usuarios, para satisfacer sus necesidades, no solo en su etapa de transición sino en la aplicación, durante los periodos subsiguientes, de ello depende el éxito del proceso.

La aplicación por primera vez, exige a los pequeños y medianos empresarios reestructurar los aspectos relacionados con el manejo y presentación de la información contable y financiera, aspecto que demanda cambios fundamentales que incorporan, entre otros, la perspectiva externa para incursionar en nuevos mercados, máxime, con el fortalecimiento que generan las telecomunicaciones, que debe convertirse en invaluable apoyo para sus procesos, como lo plantea Olivares (2005: 131) "los bajos costes de las comunicaciones a través de la red permite a las empresas con capital limitado convertirse en empresas globales en una etapa temprana de su desarrollo"; por tanto, las Pymes deben propiciar el ambiente que les permita desarrollos de negociación a escala global, aspecto que debe tenerse en cuenta desde el mismo momento de preparación obligatoria.

\section{El proceso de convergencia en Colombia}

Para alcanzar los objetivos de transparencia y uniformidad en el procesamiento de la información y la presentación de informes, se expidió la Ley 1314 de 2009, por la cual se regulan los principios y normas de contabilidad e información financiera y de aseguramiento de información, con ellas se busca que las organizaciones presenten información homogénea y de alta calidad, para que los diferentes usuarios reciban información con características tales como: comparabilidad entre las cifras de un periodo 
a otro y con competidores del mismo orden, comprensibilidad y fiabilidad de las mismas, pertinencia de los datos presentados y utilidad para la toma de decisiones.

Debe resaltarse que la implementación de las NIIF en Colombia, solo se inició en el año 2013, como respuesta a las presiones del entorno, que exigían adherirse a la tendencia de homogeneizar información, para garantizar la ya citada transparencia de las transacciones y de los informes financieros. Sin embargo, la normatividad, que clasificó a las empresas en tres grupos preparadores de información, apenas fue expedida en los dos años inmediatamente anteriores: Los grupos uno y tres, conformados por grandes empresas y microempresas, fueron regulados en el año 2012, con la expedición de los Decretos 2784 y 2706, respectivamente; en tanto que el grupo dos, al cual se asignaron las Pymes, objeto del presente análisis, se reglamentó mediante el Decreto 3022 del año 2013.

En el país se vivencian algunas situaciones que generan algún tipo de incertidumbre respecto a la convergencia, tres de ellas merecen resaltarse: La primera consiste en la marcada dependencia de las normas tributarias que bajo principios de contabilidad generalmente aceptados ha reflejado la contabilidad, aspecto que a todas luces atenta contra la transparencia de la información; al respecto Zevallos (2007) expone que los empresarios colombianos, asignaron al sistema tributario un tercer lugar entre los obstáculos para el desarrollo y Ferrer (2013: 985) plantea que la aplicación de los PCGA expresan "La influencia de la DIAN en la elección y aplicación de los principios contables y en la expedición de directrices que generan confusión en los preparadores y usuarios de la información financiera".

La segunda se genera por el gran número de planes de cuentas existentes; aspectos que producen algún tipo de recelo respecto al registro de las operaciones y genera temor e incertidumbre en los preparadores de la información en lo referente a la aplicación del nuevo marco normativo.

La tercera se manifiesta en la escasez de fuentes nacionales que contengan un estudio completo y oriente sobre el proceso de implementación de las Normas Internacionales de Información Financiera en las Pymes, diferentes a la guía de la Superintendencia de Sociedades, dado que el amplio material encontrado se enfoca fundamentalmente, a efectuar un recuento del proceso normativo que regula el accionar para los diferentes grupos de preparadores y a la actuación de los entes reguladores. Con aportes significativos se encontró la investigación desarrollada por Cardona et al sobre el nivel de preparación de medianas empresas del sector industrial en Pereira - Dos Quebradas, que concluye "La convergencia ha tenido un inicio tímido siendo por ello escasos los intentos de exploración por parte de la comunidad contable y directiva de las empresas, además de la falta de metodologías y procedimientos que permitan el desarrollo del proceso" (2013: 13).

Igualmente, un análisis sobre la implementación de las NIIF en una mediana empresa de Bogotá, encontró que en éste proceso se generaron efectos financieros y no financieros. Respecto de los primeros indicó que "pueden acarrear una desmejora en el comportamiento 
de los principales indicadores financieros" y de los segundos, señaló que generan impactos en los aspectos "operativos, económicos, administrativos, tributarios y legales" (Salazar, 2013: 407).

Es importante recalcar que el proceso de convergencia hacia las NIIF lleva implícito, replantear varios conceptos y adecuar los procesos en las organizaciones empresariales para imprimirle las características de utilidad y fiabilidad exigidas tanto en el procesamiento de la información como en la presentación de informes. En consecuencia, las empresas experimentarán diversos tipos de efectos en su estructura financiera, que dependerán en gran medida, de los criterios y políticas adoptadas, de la precisión y coherencia de las estimaciones efectuadas con el modelo de negocio.

Procesos de capacitación y preparación obligatoria. El proceso de convergencia para todos los preparadores de información, mediante cronograma implícito en el decreto reglamentario fijó los plazos y procesos a desarrollar en cada grupo, que contempló una fase de preparación obligatoria. Para las Pymes, el periodo de preparación obligatoria definido en el parágrafo 1 del artículo 3 del Decreto 3022 de 2013 se fijó entre el $1^{\circ}$ de enero y el 31 de diciembre de 2014 y durante él las empresas debieron elaborar un plan de implementación y emprender todas las acciones requeridas para el desarrollo del proceso.

Cabe destacar que la elaboración del plan de implementación exigía, que tanto contadores como empresarios, hubiesen aprehendido previa y suficientemente, las NIIF para Pymes; además, de comprender una serie de conocimientos de otras disciplinas para garantizar que la preparación fuese efectiva y permitiera su adecuado entendimiento, con el ánimo de evitar al máximo errores tanto en la definición de las políticas contables como en los procedimientos para el reconocimiento y medición de las operaciones y, con ello en la presentación y revelación de los hechos económicos al finalizar las actividades de transición, como bien lo expone la Organización Interamericana de Ciencias Económicas [OICE]:

Esta transición hacia guías de estándares comunes conlleva la necesidad de aplicar tanto los modelos de la estadística financiera como los de la matemática financiera aplicada con variaciones importantes en los usos tradicionales para reconocer la generación de valor y la valoración permanente de las entidades. (Organización Interamericana de Ciencias Económicas [OICE], s.f., justificación, párr. 2).

Lo anterior presupone que la preparación para implementar el nuevo marco normativo, debió iniciarse mucho antes de empezarse el proceso por parte de los preparadores de la información financiera; exigía a los profesionales de la contaduría emprender jornadas de capacitación, en el cual se efectuara un profundo y detallado análisis de las diferentes secciones de las NIIF para Pymes, en coherencia con la línea de negocio de la entidad, para comprender los nuevos procesos y dinámicas que al interior de la organización se derivan del cumplimiento de los nuevos estándares. Este aspecto, demanda tiempo, alto nivel de compromiso; y total conciencia de la necesidad de integrar en el proceso de preparación y capacitación a toda la organización, e incluso, a otros grupos de usuarios interesados, tales como socios o accionistas, proveedores, empleados, entre otros; 
En la responsabilidad de la formación del personal merece atención especial la participación de la empresa, dimensionar los beneficios que conlleva contribuir con la formación continua, no solo del contador sino de todo el personal de la compañía que se ve involucrado en el proceso de implementación de las NIIF, debe verse como "un valor estratégico en la empresa que permite mejorar la competitividad y posibilita la promoción social y profesional de los trabajadores" (Gutiérrez \& Lamoca, 2003:582).

Cronograma a desarrollar. Definido desde el Decreto y para el grupo dos incluye cuatro actividades claves para el desarrollo del proceso:

- Periodo de preparación obligatoria. Comprendido entre el $1^{\circ}$ de enero de 2014 hasta el 31 de diciembre de 2014, debió comenzar con la realización del plan que contemplara los términos para desarrollar actividades tales como: capacitación, asignar un responsable del proceso, asignación de recursos técnicos, humanos y financieros y todas las condiciones requeridas para iniciar la fase siguiente.

- Fecha de transición. Es la fecha que da inicio a la aplicación por primera vez, del recién adoptado marco normativo, que implica llevar información en paralelo, marco PCGA NIIF para Pymes, con inicio el 1 de enero de 2015.

Elaboración y presentación del ESFA. Consistente en la construcción del estado de situación financiera que sirve para iniciar el procesamiento y presentación de la información financiera con NIIF.

- Proceso de Transición. Se conforma por el año previo a la aplicación del nuevo marco técnico normativo, y durante el cual se deben registrar las operaciones en paralelo por los dos marcos técnicos normativos: PCGA y NIIF, con la finalidad de construir la primera información por el nuevo marco normativo y que servirá para fines estrictamente comparativos y de carácter interno; periodo que se comprende entre el $1^{\circ}$ de enero de 2015 y el 31 de diciembre de 2015.

\section{METODOLOGÍA}

El diseño metodológico corresponde a un estudio de carácter descriptivo, que se define como "aquellos que buscan únicamente describir, caracterizar situaciones o acontecimientos y sus interrelaciones, básicamente no está interesado en explicaciones, comprobar hipótesis ni hacer predicciones" (Paniagua, Pinilla \& Chajin, 2008: 68). Se utilizaron fuentes de información primarias, conformadas por encuestas aplicadas a empresarios y contadores, conocedores de la realidad que viven las Pymes de Villavicencio, contexto de estudio y, fuentes secundarias, consistentes en información de tipo legal e investigaciones recientes que abordan los procesos de aplicación de las NIIF a nivel regional, nacional y mundial.

La muestra se constituyó por 34 pequeñas y medianas empresas, en las cuales se entrevistó al empresario de mayor jerarquía (Gerente o Administrador según lo designe la empresa) y 81 Contadores Públicos, de estos, 2 cuestionarios se declararon nulos debido a la gran cantidad de preguntas que no obtuvieron respuesta. 
Para la recolección de información primaria, se utilizó un formulario tipo encuesta, elaborado con alternativa de respuesta cerrada. El procesamiento y análisis de la información se realizó con el apoyo de dos sistemas informáticos: Excel de Microsoft 2010, para la organización de la información y el software IBM SPSS Statistics versión19, para el procesamiento y generación de los índices estadísticos.

\section{RESULTADOS}

En el estudio se determinó que las actividades a realizar en la aplicación por primera vez de las NIIF, deben agruparse en tres etapas: preparación, desarrollo y resultados, como se muestra en la figura 1, igualmente, cada etapa se relacionó con un nivel o grado de avance de los procesos, con base en dos parámetros:

- Fase en que se encuentra el proceso de implementación.

- Cumplimiento del cronograma previsto por el Decreto 3022 de 2013, aplicable al grupo dos.

En tal sentido, la etapa de preparación se relacionó con una ejecución del proceso en nivel bajo, la etapa de desarrollo con un nivel medio y la de resultados, con un nivel alto.

Preparación. En atención a que esta etapa se considera fundamental tanto para el proceso de implementación de las NIIF como para el proyecto de investigación que origina el presente artículo, se encontró, que previo al desarrollo del proceso, las empresas deben realizar una serie de actividades preliminares clave, que no fueron contempladas en la guía práctica de la Superintendencia, por ello se subdividió en dos fases: previa e inicio. La fase previa se conformó con actividades consistentes en: capacitar al personal, apropiar recursos, revisar los procesos y elaborar el plan de acción, en tanto que a la fase de inicio se asignan las actividades relacionadas con la organización de la información de acuerdo con los preceptos de las NIIF para Pymes (Figura 1). Para establecer el estado de avance de las entidades, ésta fase se identificó con un nivel bajo de preparación.

Desarrollo. Esta fase se encarga de realizar las actividades concernientes a la obtención de la información para elaborar la hoja de trabajo del ESFA. Proceso que requiere de un análisis minucioso de la actividad económica, la información financiera elaborada bajo PCGA y las NIIF para Pymes con el fin de determinar las partidas que cumplen con la condición de activo y pasivo. Para efectos de determinar el grado de avance logrado por las entidades, ésta fase se le asignó un nivel medio de realización.

Resultados. En esta etapa se lleva a cabo la culminación del ESFA y, se determinan los efectos en términos fiscales con el cálculo y registro del impuesto diferido. De igual manera, se realiza un conjunto de reportes que informan sobre el impacto financiero de la conversión a estándares internacionales; sobresalen entre ellos, la conciliación de los resultados del ejercicio y patrimonio de los PCGA a las NIIF para Pymes y el informe de conversión. Del mismo modo, se da inicio al proceso de transición, en el cual se registran 
las operaciones por los dos marcos normativos. La Pyme que ha llegado a ésta etapa se la identifica con un nivel alto de desarrollo.

Posterior a la figura 1, se presentan los resultados en torno a dos aspectos: caracterización de la muestra y diagnóstico realizado sobre el nivel de preparación de las Pymes analizadas.

\section{Figura 2. Etapas del Proceso de Implementación de NIIF en las Pymes.}

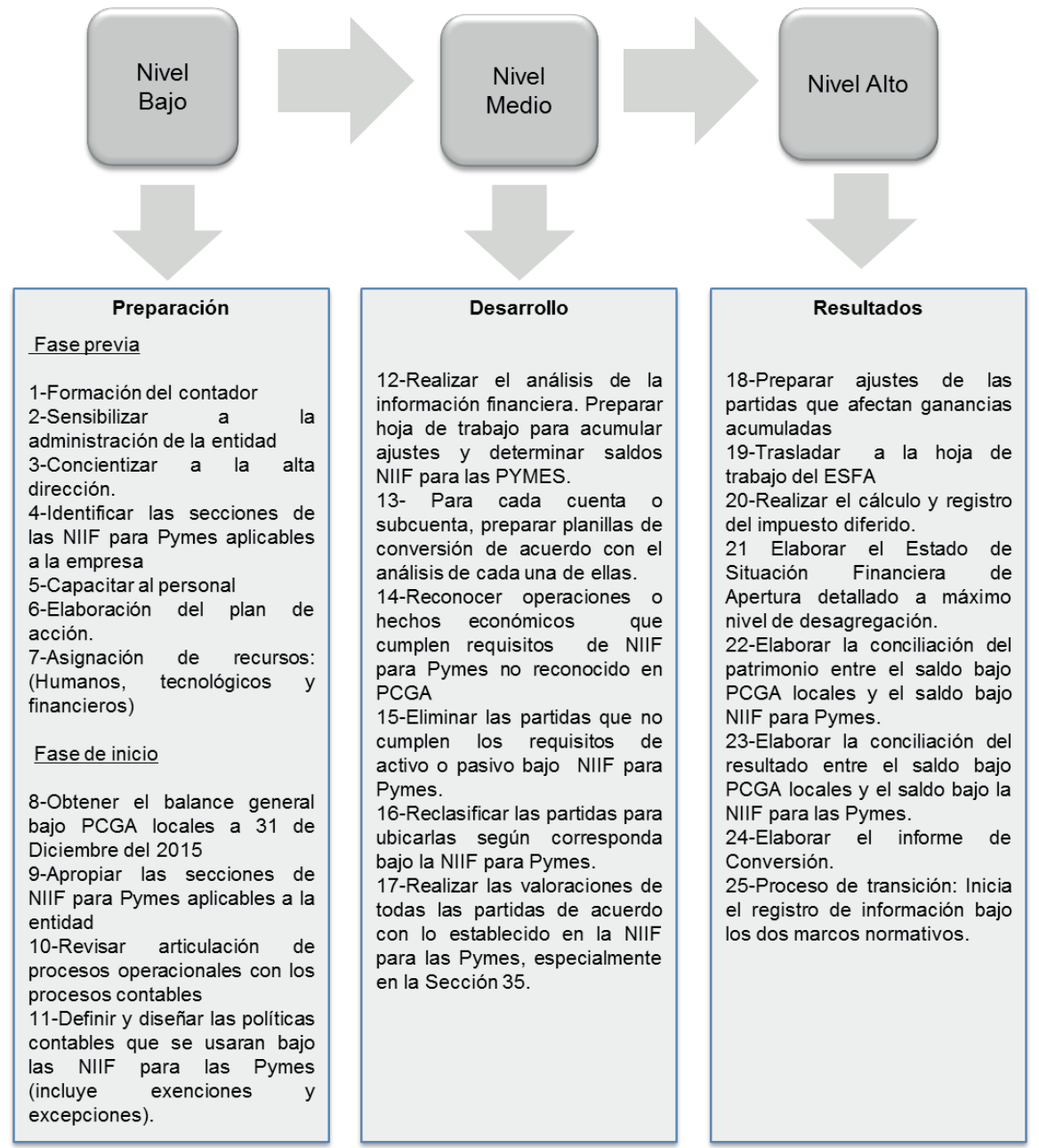

Fuente: Adaptado de la Guía práctica para elaborar el Estado de Situación Financiera de Apertura bajo NIIF PYMES Superintendencia de Sociedades. (2014, pp.37-38).

\section{Caracterización de la muestra}

Corresponde a la descripción de los principales rasgos de las Pymes de Villavicencio que conforman la muestra, con base en los siete aspectos que se relacionan en la tabla 2: 
Como datos relevantes, los resultados indicaron que las Pymes son relativamente jóvenes, el $44 \%$ de ellas tiene menos de cinco años de constituidas, el 18\% menos de diez años y solo el 38\% se conformó hace más de diez años. La gran mayoría (68\%) son pequeñas empresas, el $24 \%$ se alcanzan los parámetros para catalogarse como medianas y el $8 \%$ restante, al revisar su nivel de activos y número de empleados, no alcanzó a categorizarse como Pymes.

Referente a la implementación de las NIIF, solo el 40\% de las pequeñas y medianas empresas dio inicio a los procesos de implementación de NIIF y el restante $60 \%$ no ha empezado.

Con relación a la capacitación en NIIF, el 16\% no ha efectuado ningún tipo de capacitación, el 36\% ha desarrollado este tipo de procesos con intensidad inferior a 20 horas, el 24\% le dedicó entre 21 y 50 horas y solo el restante $24 \%$ utilizó más de 50 horas.

En relación con la inversión asignada, el 20\% no ha proporcionado recursos para el desarrollo de procesos relacionados con la capacitación en NIIF, el 29\% le ha invertido menos de dos millones de pesos, el 20\% utilizó recursos en un rango de dos a cinco millones, el 6\% le adjudicó entre 5 y 10 millones y el restante $25 \%$ asignó más de 10 millones.

Tabla 2. Caracterización de la muestra

\begin{tabular}{lcc}
\hline Características & Pymes & $\%$ \\
\hline & Menos de 2 & 10 \\
Edad (Años) & 2 a 5 & 34 \\
& 5 a 9 & 18 \\
& 10 a 15 & 29 \\
\hline Tamaño de la Pyme & Más de 15 & 9 \\
\hline Forma de realizar la capacitación en & Pequeña & 68 \\
NIIF & Mediana & 24 \\
& Interna (Realizada por el Contador) & 53 \\
& & 31
\end{tabular}




\begin{tabular}{lcc}
\hline Características & Rasgo & $\%$ \\
\hline & Ninguna & 16 \\
& Menos de 20 & 36 \\
Horas de capacitación en NIIF & 21 a 50 & 24 \\
& 51 -100 & 12 \\
& 101 a 200 & 12 \\
& Más de 200 & 0 \\
Recursos invertidos en el proceso de & Sin inversión & 20 \\
implementación de NIIF & Menos de dos (2) millones & 29 \\
Desarrollo de los procesos de & 2 a 5 millones & 20 \\
implementación NIIF & 5 a 10 millones & 6 \\
\hline & No ha iniciado & 60 \\
Fase del proceso* & En desarrollo & 40 \\
\hline
\end{tabular}

Notas: * Solo se aplicó a las empresas que iniciaron el proceso

Fuente: Elaboración propia

Adicionalmente, los resultados reflejan que la mayoría de las Pymes que se capacitaron, lo hicieron en forma interna y el encargado de realizar dicha capacitación fue el contador. Como dato concluyente se encontró que el 71\% de las Pymes que dio inicio al proceso se encuentra apenas en la primera fase previa de la etapa de preparación.

\section{Nivel de preparación en NIIF en las Pymes de Villavicencio}

Se describen en la Tabla 3, el estado de las siete actividades de la fase previa desarrolladas y el grado de desarrollo (\%) en que se encuentran.

Los datos evidenciaron un nivel bajo de desarrollo en cinco de las siete actividades de la fase previa; por lo cual vale la pena resaltar, que del total de las actividades, las cuatro primeras son responsabilidad del contador: Su propio proceso de formación, sensibilizar a la Junta Directiva, concientizar a la alta dirección, identificar la normatividad y establecer las secciones de las NIIF para Pymes aplicables a la línea de negocio; el desarrollo de las tres restantes le corresponde a la entidad, en cabeza de su administración. 
Referente a la capacitación del contador (actividad 1), la tabla 3 indica que ésta cubre solo una tercera parte de conocimiento del marco normativo de las NIIF, por lo cual se le relacionó con un nivel bajo. Respecto de las actividades dos y tres estas alcanzan un grado medio de realización, $41 \%$ y $43 \%$ de realización, solo en el $33 \%$ de las Pymes se han identificado las secciones de las NIIF que le son aplicables.

El análisis de los datos presentados indicó que las actividades señaladas como responsabilidad del Contador alcanzaron el mayor grado de realización, en tanto que las actividades correspondientes al accionar de la administración, evidenciaron los menores grados de avance, sobresale, entre las últimas, la asignación de recursos con el menor índice de ejecución en todas las actividades de la fase.

\section{Tabla 3. Nivel de preparación para implementar NIIF en las Pymes de Villavicencio}

\begin{tabular}{clccc}
\hline No. & \multicolumn{1}{c}{ Actividad } & Nivel & $\begin{array}{c}\text { Grado de realiza- } \\
\text { ción promedio (\%) }\end{array}$ & $\begin{array}{c}\text { Desviación } \\
\text { típica (\%) }\end{array}$ \\
\hline 1 & Formación del contador & Bajo & 33 & 12 \\
2 & Sensibilización a la Junta Directiva & Medio & 41 & 17 \\
3 & Concientización Alta Dirección & Medio & 43 & 18 \\
4 & $\begin{array}{l}\text { Identificación de las secciones de NIIF } \\
\text { para Pymes aplicables }\end{array}$ & Bajo & 33 & 15 \\
6 & Capacitación del personal & Bajo & 32 & 13 \\
7 & Asignación de recursos al proceso & Bajo & 25 & 1 \\
\hline
\end{tabular}

Fuente: Elaboración propia.

\section{Discusión}

El proceso de convergencia en Colombia, se ha constituido en gran reto para las Pymes, la normatividad que lo rige, es de muy reciente expedición y, aunque desde una década atrás se vislumbraba el ingreso de las NIIF al país, hubo poca difusión y preparación al respecto, a todo nivel, estatal, empresarial, académica y de gremios y la escasa difusión alcanzada, se direccionó a las grandes empresas: cotizantes en bolsa y empresas de interés público. Cabe resaltar que apenas en el año 2009 el organismo encargado de su expedición, Consejo de Normas Internacionales de Contabilidad (IASB por sus siglas en inglés) emitió la Norma Internacional de Información Financiera para Pymes, su aplicación a nivel mundial es tan reciente como su normatividad y los estudios que dan cuenta de su aplicación son relativamente escasos, situación que no es ajena al País. 
El panorama contable de las Pymes colombianas se agrava con la tradicional afectación de la información contable y financiera nacional, por las normas fiscales, que ha condicionado la perspectiva de utilidad de dicha información en el pequeño y mediano empresario colombiano, dirigiéndola, casi que exclusivamente, hacia efectos fiscales; no en vano, el sistema tributario fue señalado como uno de los obstáculos que afectan el desempeño de las empresas en Colombia (Zevallos, 2007), y que también es señalado por Ferrer (2013). Dado, que la falta de tiempo, se indicó por los Contadores Públicos, como una de las principales causas para el retraso en las actividades del proceso de implementación, también vale la pena cuestionarse acerca del nivel de incidencia de la excesiva carga tributaria de las empresas, por tanto, en el accionar profesional del Contador, en aspectos como: la calidad de la información, de lo adecuado de los honorarios en las pequeñas y medianas empresas. De ahí la importancia de una real separación de la normativa fiscal y contable. Todo ello lleva a otra pregunta ¿Puede la estructura financiera de las Pymes soportar el impacto financiero de la separación?

Respecto de la preparación, los análisis realizados confirman la importancia de la fase previa en el proceso de elaboración del ESFA y el proceso de transición, se resalta la importancia de cuatro aspectos: formación del Contador Público, preparación propiamente dicha de la Pymes, inversión de recursos y utilidad de la información; cada una de ellas evidencia una relevancia de tal magnitud, que se constituye en factor determinante en el grado o nivel de desarrollo en la implementación de las NIIF en las Pymes.

Merece resaltarse, las Pymes que por sí solas ya evidencian múltiples limitaciones, se insertaron en el proceso de implementación de las NIIF; proceso que es responsabilidad de la administración y, que en su gran mayoría le ha sido asignado, en buena parte, a los contadores, para lo cual deben demostrar su aptitud profesional.

\section{CONCLUSIONES}

En este documento se resalta la importancia de la fase de preparación en el proceso de elaboración del ESFA y el proceso de transición. Dicha fase, adicionada por la autora a la guía de actividades para llevar a cabo la implementación de las NIIF para Pymes, se conforma por cuatro subprocesos: formación del Contador Público, preparación propiamente dicha de la Pymes, inversión de recursos y utilidad de la información, cada uno de ellos se convierte en factor determinante para el desarrollo del proceso en éste tipo de entidades; que además, se constituyen en parte importante de la problemática que limita o retrasa el avance del proceso, por lo cual debe analizarse en el contexto de cada organización.

Respecto al proceso de preparación en NIIF en las Pymes de la ciudad de Villavicencio analizadas, se detectó que la mayoría, de las Pymes revisadas no ha iniciado el proceso de implementación de las NIIF ad portas de vencerse los plazos y la minoría, que dio inicio al proceso de implementación, evidenció un bajo grado de avance en las actividades, dado que solo han realizado lo concerniente a la fase previa del proceso de la aplicación por primera vez. 
Como aspecto concluyente, se evidenció que el bajo nivel de preparación,indudablemente retrasó el proceso, aspecto en el cual comparten responsabilidad tanto los contadores como la administración; los primeros por su bajo nivel de capacitación, los segundos debido a que no mostraron el interés requerido ni asignaron los recursos necesarios.

\section{REFERENCIAS}

(1) CARDONA H, VELANDIA T \& GIRALDO R. (2013). Nivel de preparación de las Medianas Empresas del Sector Industrial Pereira - Dosquebradas para adoptar Normas Internacionales de Contabilidad, Revista Curn, p. 13. Recuperado en de http://revistas.curnvirtual.edu.co/journals/index.php/aglala/article/view/349

(2) COMISIÓN ECONÓMICA PARA AMÉRICA LATINA Y EL CARIBE Y ORGANIZACIÓN PARA LA COOPERACIÓN Y EL DESARROLLO ECONÓMICOS - CEPAL.-OCDE. (2013.) Perspectivas Económicas de América Latina 2013, p. 52. Recuperado de: http://www.cepal.org/es/publicaciones/1463-perspectivas-economicas-de-america-latina-2013-politicas-de-pymes-para-el-cambio

(3) COLOMBIA. Ministerio de Comercio, Industria y Turismo. 2013. Decreto 3022 de 2013, por el cual se reglamenta la Ley 1314 de2009 sobre el marco técnico normativo de información financiera para los preparadores de información del grupo 2 Diario Oficial, 49.01627 de diciembre de 2013. Recuperado de: http://www. icbf.gov.co/cargues/avance/docs/decreto_3022_2013.htm

(4) COLOMBIA. Presidencia de la República. (2012). Decreto 2706 de 2012, por el cual se reglamenta la Ley 1314 de2009 sobre el marco técnico normativo de información financiera para las microempresas. Diario Oficial, 48.657, 28 de diciembre de 2012. Disponible en: http://www.alcaldiabogota.gov.co/sisjur/normas/Norma1.jsp?i=51148

(5) COLOMBIA. Ministerio de Comercio, Industria y Turismo. (2012). Decreto 2784 de 2012, por el cual se reglamenta la Ley 1314 de 2009 sobre el marco técnico normativo para los preparadores de información financiera que conforman el Grupo 1. Diario Oficial, 48.658, 29 de diciembre de 2012. Disponible en: http:// www.minhacienda.gov.co/portal/page/portal/HomeMinhacienda/regulacionfinanciera/Decretos/2012/ DECRETO\%202784\%20DE\%202012.pdf 
(6) COLOMBIA. Congreso de la República. (2009) Ley 1314 de 2009, por la cual se regulan los principios y normas de contabilidad e información financiera y de aseguramiento de información aceptados en Colombia, se señalan las autoridades competentes, el procedimiento para su expedición y se determinan las entidades responsables de vigilar su cumplimiento. Diario Oficial, 47.409 de Julio13 de 2009, disponible en http://www.alcaldiabogota. gov.co/sisjur/normas/Norma1.jsp?i=36833

(7) COLOMBIA. Congreso de la República. (2004) Ley 905 de 2004, Por medio de la cual se modifica la Ley 590 de 2000 sobre promoción del desarrollo de la micro, pequeña y mediana empresa colombiana y se dictan otras disposiciones. Diario Oficial No. 45.628 de 2 de agosto de 2004. Recuperado de http://www.secretariasenado.gov.co/senado/basedoc/ley_0905_2004.html

(8) COLOMBIA. Congreso de la República. (1990). Ley 43 de 1990, por la cual se adiciona la Ley 145 de 1960 , reglamentaria de la profesión de contador público y se dictan otras disposiciones. Diario Oficial, 39.602,13 de diciembre de 1990. Recuperado de: http://ftp.camara.gov.co/camara/basedoc/ley/1990/ley_0043_1990.html

(9) FERRER, A. (2013). Análisis del proceso de convergencia a normas internacionales de contabilidad e información financiera desde los factores intrínsecos al sistema contable de Colombia. Pontificia Universidad Javeriana, Cuadernos de Contabilidad, 14 (36), 971-1007. Recuperado en Noviembre 15 de 2014 de http:// revistas.javeriana.edu.co/index.php/cuacont/article/viewFile/7910/6293

(10) GONZALO, A. J.A. 2004. Normas IASB: La primera vez. Universia Business Review. Número 1, p. 110. Recuperado en diciembre 23 de 2014 de: http://www.redalyc.org/articulo.oa?id=43300110

(11) GUTIÉRREZ, J.R \& LAMOCA, M. (2002). Necesidad e importancia de la formación para el puesto de trabajo. La acción formativa de la unión sindical de comisiones obreras de Palencia (1997-2000). Revista Universitaria de Ciencias del Trabajo, (3), 577-604. Recuperado en Noviembre 15 de 2014 de https://uvadoc.uva.es/ bitstream/10324/11224/1/RevistaUniversitariadeCienciasdelTrabajo-2002-2003-n\%C2\%BA\%203-4-Necesidadeimportanciadelaformacion.pdf

(12) Mejía, E. (2005). Investigación Contable. Revista Internacional Legis de Contabilidad y Auditoría, (24), 135174. Recuperado en Noviembre 15 de 2014 de http://eutimiomejia.com/articulos/introduccionpensamientocontableRichardMattessich.pdf

(13) CONSEJO DE NORMAS INTERNACIONALES DE CONTABILIDAD - IASB. (2009) NIIF para las PYMES. Editado por IASCF 245 p.

(14) OLIVARES, A. (s.f.). La globalización y la internacionalización de la empresa: ¿Es necesario un nuevo paradigma? Icesi Estudios gerenciales, (96), 127-137. Recuperado en noviembre 13 de 2014 de http://www.icesi. edu.co/revistas/index.php/estudios_gerenciales/article/view/167/165

(15) ORGANIZACIÓN INTERAMERICANA DE CIENCIAS ECONÓMICAS OICE. (s.f.). OICE: XBRL y Finanzas Corporativas y Matemáticas Financieras para el entendimiento de las NIIF. Bogotá, Colombia, párr. 2 Recuperado en diciembre 1 de 2014 de http://www.oice.org/finanzas1/finanzas.html

(16) PANIAGUA, F.R, PINILLA, M.P, CHAJÍN, F.M. (2008). Metodología de la Investigación. Editorial Uniautonoma, Barranquilla. p. 68.

(17) SAAVEDRA, G., M.L. (2012). Una propuesta para la determinación de la competitividad en la pyme latinoamericana Pensamiento \& Gestión, núm. 33, julio-diciembre, p. 98- Universidad del Norte, Barranquilla, Colombia. Recuperado en Noviembre 15 de 2014 de: http://www.redalyc.org/pdf/646/64624867005.pdf

(18) SALAZAR, E. (2013.) Efectos de la implementación de las NIIF para las pymes en una mediana empresa ubicada en la ciudad de Bogotá. Pontificia Universidad Javeriana. Cuadernos de contabilidad 14 (35), p. 407. Recuperado en Enero 10 de 2015 de http://www.scielo.org.co/pdf/cuco/v14n35/v14n35a03.pdf 
(19) SÁNCHEZ, A \& GIRALDO N. (2008) Las necesidades de las pyme - pequeñas y medianas empresas - y el sistema de información contable y financiero como una estrategia para atenderlas. Cuadernos de Contabilidad, 5 (25), 421-464. Recuperado en Noviembre 15 de 2014 de http://revistas.javeriana.edu.co/index.php/ cuacont/article/view/3232/2456

(20) SUPERINTENDENCIA DE SOCIEDADES. (2014). Guía práctica para elaborar el Estado de Situación Financiera de Apertura bajo NIIF PYMES. Recuperado en Enero de 2015 de http://www.supersociedades.gov.co/asuntos-economicos-y-contables/procesos-de-convergencia-niifs/material-pedagogico/guia-aplicacion-primera-vez-grupo2/Documents/Guia-practica-05082014.pdf

(21) TÚA, P. J. 2009. Contabilidad y desarrollo económico. El papel de los modelos contables de predicción. Especial referencia a las NIC'S. En Facultad de Ciencias Administrativas y Económica, Universidad Icesi, Revista Estudios Gerenciales (Presidencia), Análisis y propuestas creativas ante los retos del nuevo entorno empresarial. Simposio llevado a cabo en la Universidad ICESI, Cali, Colombia.

(22) TUA, P. J. (1988). Evolución del concepto de contabilidad a través de sus definiciones. Contaduría Universidad de Antioquia, (13), 9-74. Recuperado en Enero 14 de 2015 de http://aprendeenlinea.udea.edu.co/revistas/ index.php/cont/article/view/24995/20322

(23) VÁSQUEZ, B. R y FRANCO, F. W (2014) Aplicación por primera vez de las NIIF. Legis Editores S.A. Primera Edición. Bogotá Colombia. 3-5

(24) ZEVALLOS, E. (2007). Restricciones del entorno a la competitividad empresarial en América Latina- Documento de trabajo FUNDES internacional, San José de Costa Rica., 1 - 46. 